\title{
Validación de la Escala de Aceptabilidad de la Violencia de Pareja Contra la Mujer (A-IPVAW) en Población Ecuatoriana
}

\author{
Validation of the Acceptability of Intimate Partner Violence Against Women (A- \\ IPVAW) Scale in Ecuadorian Population
}

\author{
Aída M. Villagrán ${ }^{1}$, Manuel Martín-Fernández ${ }^{2}$, Enrique Gracia ${ }^{3}$ y Marisol Lila ${ }^{4}$
}

\begin{abstract}
Resumen
Ecuador es uno de los países latinoamericanos con una mayor prevalencia de violencia de pareja contra la mujer (VPM), un problema social y de salud pública que tiene graves consecuencias para el bienestar de las mujeres. Las actitudes de aceptabilidad hacia la VPM son un factor esencial para comprender mejor este problema y desarrollar iniciativas de prevención e intervención eficaces. En este estudio se presenta la validación de la escala de actitudes de aceptabilidad de la VPM española (A-IPVAW) al contexto sociocultural ecuatoriano en dos muestras de 992 y de 346 participantes. Los resultados mostraron que esta medida posee una buena consistencia interna, que es invariante entre géneros, que sus ítems tienen una baja carga de deseabilidad social, y que se relaciona con otros constructos actitudinales teóricamente relevantes. Estos hallazgos proporcionan evidencia de que la escala A-IPVAW es un instrumento adecuado para evaluar la aceptabilidad de la VPM en Ecuador.
\end{abstract}

Palabras clave: violencia de pareja contra la mujer, aceptabilidad, actitudes, medición, Ecuador

\begin{abstract}
Ecuador is one of the Latin American countries with the highest prevalence of intimate partner violence against women (IPVAW), a social and public health issue with severe consequences for the well-being of women. Attitudes of acceptability towards IPVAW are a key factor to understand this problem and developing effective prevention and intervention initiatives. In this study, we present the validation of the Spanish acceptability of the IPVAW Scale (A-IPVAW) to the Ecuadorian sociocultural context in two samples of 992 and 346 participants. Results showed that this measure has a good internal consistency, that is invariant across gender, that their items present low loadings of social desirability, and that it is related to other theoretically relevant attitudinal constructs. These findings provide evidence that the A-IPVAW scale is an adequate measure to assess the acceptability of IPVAW in Ecuador.
\end{abstract}

Keywords: intimate partner violence against women, acceptability, attitudes, measurement, Ecuador

Agradecimientos: Esta investigación ha sido financiada por FEDER/Ministerio de Ciencia, Innovación y Universidades - Agencia Estatal de Investigación/ Proyecto (PSI2017-84764-P).

\footnotetext{
${ }^{1}$ Máster en Intervención Psicológica en Ámbitos Sociales e Investigadora Predoctoral. Departamento de Psicología Social, Universidad de Valencia. Correo: aivive@alumni.uv.es. ORCID: 0000-0002-1195-2029

${ }^{2}$ Doctor en Psicología. Profesor Ayudante Doctor en la Universidad Autónoma de Madrid. Calle Iván Pavlov, 6, Departamento de Psicología Social y Metodología, Universidad Autónoma de Madrid 28049, Madrid, España. Tel.: +34 914974061. Correo: manuel.martinfernandez@uam.es. ORCID: 0000-0003-3606-3559. (Autor de correspondencia)

${ }^{3}$ Doctor en Psicología y Catedrático de la Universidad de Valencia. Departamento de Psicología Social, Universidad de Valencia, España. Correo: enrique.gracia@uv.es. ORCID: 0000-0003-0514-2983

${ }^{4}$ Doctora en Psicología y Catedrática de la Universidad de Valencia. Departamento de Psicología Social, Universidad de Valencia, España. Correo: marisol.lila@uv.es. ORCID: 0000-0002-0522-7461
}

Revista Iberoamericana de Diagnóstico y Evaluación - e Avaliação Psicológica. RIDEP · No62 · Vol.1 · 67-81 · 2022 


\section{Introducción}

La violencia de pareja contra la mujer (VPM) es la forma más común de violencia que sufren las mujeres (Devries et al., 2013; Organización Mundial de la Salud [OMS], 2013; Stöckl et al., 2013). Se trata de un fenómeno de proporciones globales, un problema social y de salud pública que tiene graves consecuencias para el bienestar tanto físico como psicológico de las mujeres (Martín-Fernández et al., 2019, 2020; OMS, 2013; Vilariño et al., 2018). A nivel mundial, mientras que los hombres tienen mayor probabilidad que las mujeres de sufrir violencia en conflictos armados y actividades delictivas, las mujeres tienen mayor riesgo de ser víctimas de violencia perpetrada por personas cercanas a su entorno, como sus parejas (Guedes et al., 2014). Según la OMS (2013), la prevalencia mundial de VPM oscila entre un $16.3 \%$ y $41.7 \%$, dependiendo de la región. Concretamente, en Latinoamérica Andina la prevalencia de VPM es de 40.6\%. En Latinoamérica también se encuentran más de la mitad de los países con mayores tasas de femicidio a nivel mundial (Saccomano, 2017). En particular, Ecuador es uno de los países latinoamericanos con una mayor proporción de casos de VPM, donde el $42.8 \%$ de las mujeres mayores de quince años indica haber sido víctima de VPM en algún momento de su vida (Instituto Nacional de Estadísticas y Censos, 2019) y donde el $25 \%$ de las mujeres afirma que la VPM ocurre de manera muy frecuente (Camacho, 2014). Todo ello pone de manifiesto que la VPM es una problemática profundamente arraigada en el contexto sociocultural ecuatoriano.

Con el fin de comprender los factores individuales y sociales que contribuyen a explicar la prevalencia de VPM, el estudio de las actitudes públicas hacia la VPM se ha convertido en un tema central, debido a que dan forma al entorno sociocultural donde la violencia tiene lugar y pueden contribuir a incrementar o reducir su ocurrencia (Campbell \& Manganello, 2006; Gracia et al., 2020). Investigaciones previas subrayan la estrecha relación que existe entre las actitudes públicas hacia la VPM y las respuestas individuales, comunitarias e institucionales frente a la VPM (Gracia et al., 2014, 2018; Lila et al., 2013; Sánchez-Prada et al., 2019). Además, las actitudes públicas hacia la VPM tienden a transmitirse entre generaciones, pudiendo incidir en el mantenimiento y perpetuación de este tipo de violencia (Barker et al., 2011; Fleming et al., 2015). Las actitudes de aceptabilidad de la VPM son por tanto un factor de riesgo clave para la perpetración de VPM. Estas actitudes pueden contribuir a que las personas perciban la VPM como una conducta normativa, justificando la violencia y aumentando el riesgo de que los hombres cometan VPM (Coop et al.bola, 2019; Fleming et al., 2015; Gracia et al., 2015; MartínFernández et al., 2018; Romero-Martinez et al., 2019; Waltermaurer, 2012). Las actitudes de aceptabilidad de la VPM también pueden ser asimismo un obstáculo para que las mujeres víctimas de VPM soliciten ayuda y pueden disuadir a su círculo social de proporcionársela (Flood \& Pease, 2009; Gracia, 2014; Gracia et al., 2011; Kogut, 2011; Waltermaurer, 2012). Distintos estudios ponen además de manifiesto que los niveles de aceptabilidad de la VPM son de mayor prevalencia entre los agresores de este tipo de violencia (Gracia et al., 2015; Herrero et al., 2020; Martín-Fernández et al., 2018). Por todo ello, las actitudes de aceptabilidad de la VPM son uno de los principales objetivos de intervención de los programas y políticas sociales dirigidos a la prevención de la VPM (García-Moreno et al., 2015; Gracia, Rodríguez et al., 2020; Heise, 2011; Jewkes et al., 2015).

\section{El presente estudio}

A pesar de la relevancia que tienen las actitudes hacia la VPM, los datos sobre la aceptabilidad de la VPM en Ecuador son escasos $\mathrm{y}$ provienen principalmente de encuestas sociodemográficas que incluyen algunas preguntas o ítems únicos (Bott et al., 2013; Bucheli \& Rossi, 2016; Safranoff, 2017; Tausch, 2019). Se hace indispensable, por tanto, contar con instrumentos fiables y válidos que permitan evaluar de manera consistente la aceptabilidad de la VPM en el contexto sociocultural de Ecuador.

El objetivo del presente estudio es validar la escala española de aceptabilidad de la VPM (AIPVAW; Martín-Fernández, Gracia, Marco et al., 2018) en población ecuatoriana. Este instrumento cuenta con unas propiedades psicométricas adecuadas (i.e., fiabilidad y validez) en el 
contexto sociocultural español. No obstante, se desconoce si la escala A-IPVAW puede evaluar de manera adecuada este tipo de actitudes en el contexto sociocultural de Ecuador. Con este fin, se examinará la estructura latente, la consistencia interna, el efecto de la deseabilidad social de esta escala, así como su invarianza factorial entre géneros. Además, se analizarán las evidencias de validez de la escala basada en su relación con otros constructos teóricamente relevantes, como son las actitudes de culpabilización de la víctima en casos de VPM, la gravedad percibida de la VPM, y el sexismo hostil y benevolente (Gracia et al., 2008; Gracia et al., 2018; Lila et al., 2010, 2013; Martín-Fernández et al., 2018; SánchezHernández et al., 2020; Valor-Segura et al., 2011) y se explorará la relación entre la escala AIPVAW y diferentes variables sociodemográficas como el género, el nivel educativo y la edad (Carlson \& Worden, 2005; Fincham et al., 2008; Flood \& Pease, 2009; Gracia et al., 2015; Gracia \& Tomás, 2014; López-Cepero Borrego et al., 2015; Vidal-Fernández \& Megías, 2014).

\section{Método}

\section{Participantes}

En este estudio se utilizaron dos muestras. La primera estuvo compuesta por 992 participantes ecuatorianos $(53.9 \%$ mujeres $)$ de edades comprendidas entre los 18 y los 72 años $\left(M_{\text {edad }}=\right.$ $\left.26.57, D T_{\text {edad }}=9.68\right)$. De ellos, el $2.5 \%$ completó los estudios básicos, el $28.8 \%$ los estudios secundarios, el $37.9 \%$ cursaba estudios universitarios y el $30.8 \%$ poseía formación universitaria. La segunda muestra estuvo compuesta por 346 estudiantes universitarios de Ecuador (53.5\% mujeres), de edades entre los 18 y los 25 años $\left(M_{\text {edad }}=20.54, D T_{\text {edad }}=1.96\right)$. Ambas muestras fueron reclutadas a través de un muestreo en línea no probabilístico de tipo bola de nieve.

\section{Instrumentos}

Actitudes de aceptabilidad de la VPM (AIPVAW; Martín-Fernández et al., 2018). La escala A-IPVAW consta de 20 ítems donde los participantes han de evaluar cómo de aceptables son una serie de comportamientos relacionados con la VPM (e.g., "Pienso que es aceptable para un hombre evitar que su pareja vea a amigos o familiares"). El formato de respuesta de los ítems es una escala Likert de tres puntos $(0=$ "Nada aceptable", 2="Aceptable"). Esta escala ha sido validada en población general española y se ha relacionado previamente con sexismo ambivalente, gravedad percibida y disposición a intervenir en casos de VPM (Gracia et al., 2018; Martín-Fernández et al., 2018). Los ítems de la versión adaptada al contexto sociocultural de Ecuador pueden encontrarse en el Anexo.

Actitudes de culpabilización de la víctima en casos de VPM (VB-IPVAW; Martín-Fernández et al., 2018). La escala VB-IPVAW consta de 12 ítems que evalúan las actitudes de culpabilización a la víctima (e.g., "Los hombres cambiarían la conducta violenta hacia su pareja si ellas fueran más obedientes"). El formato de respuesta de los ítems fue una escala Likert de cuatro puntos (1="muy en desacuerdo", 4="muy de acuerdo"). Esta escala ha sido validada en población general española y también en población ecuatoriana (Villagrán et al., 2020). La escala VB-IPVAW se ha relacionado previamente con la gravedad percibida y la disposición a intervenir en casos de VPM (Gracia et al., 2018; Martín-Fernández, Gracia \& Lila., 2018). La escala mostró una buena consistencia interna en la primera muestra de este estudio $(\alpha=.91, \omega=.92)$.

Gravedad percibida de la VPM (PS-IPVAW; Gracia et al., 2008). Esta escala presenta ocho escenarios de VPM (e.g., "En una discusión, un hombre pega a su pareja y después le pide perdón"), cuya gravedad fue evaluada por los participantes en una escala de respuesta tipo Likert de diez puntos (1="no es grave en absoluto", 10="extremadamente grave"). La escala PS-IPVAW ha sido previamente validada en población general española, con policías y con hombres penados por VPM, presentando propiedades psicométricas aceptables. Esta escala se ha relacionado con el sexismo, la empatía y responsabilidad personal (Gracia et al., 2014; Lila et al., 2013, 2014). La escala mostró una buena consistencia en la primera muestra de este estudio $\left(\alpha=.96\right.$ y $\left.\omega_{\text {total }}=.96\right)$.

Inventario de sexismo ambivalente (ASI; Glick \& Fiske, 1996; adaptación española de Expósito et al., 1998). Este instrumento consta de 22 ítems con un formato respuesta tipo Likert de 
cinco puntos $(0=$ "totalmente en desacuerdo", 5="totalmente de acuerdo"). Esta escala está compuesta por dos factores: sexismo hostil y sexismo benevolente. El primero se relaciona con una actitud de prejuicio y discriminación hacia la mujer basada en su supuesta inferioridad y subordinación en relación al hombre (e.g., "las mujeres feministas están haciendo demandas completamente irracionales a los hombres"). El segundo factor se define como una actitud que considera a las mujeres de forma estereotipada y limitada a ciertos roles con un tono afectivo positivo (e.g., "el hombre está incompleto sin la mujer"). Este inventario se ha relacionado con la atribución de responsabilidad y la aceptabilidad de VPM (Lila et al., 2013; Lila et al., 2014; MartínFernández, Gracia \& Lila, 2018; SánchezHernández et al., 2020). La consistencia interna de la escala completa $\left(\omega_{\text {total }}=.88\right)$ y de ambas subescalas fue buena en la primera muestra $(\alpha=.85$ y $\omega_{\text {total }}=.86$ para sexismo hostil, $\alpha=.77$ y $\omega_{\text {total }}=.76$ para seximo benevolente).

Manejo de impresiones. Para medir esta variable se utilizó la subescala manejo de impresiones de la versión breve del inventario balanceado de respuesta deseable (BIDR-16; Hart et al., 2015). Esta subescala consta de ocho ítems con un formato de respuesta tipo Likert de cinco puntos (1="muy en desacuerdo", 5="muy de acuerdo") que evalúan la tendencia de los participantes a sobreestimar su auto-descripción para crear una imagen socialmente deseable (e.g., "Nunca digo malas palabras"). La consistencia interna de esta medida fue buena $(\alpha=.82 \mathrm{y}$ $\omega_{\text {total }}=.82$ ).

\section{Procedimiento}

Se diseñó un formulario en línea para obtener los datos. Este formulario incluía la escala AIPVAW, la escala VB-IPVAW, la escala PSIPVAW, el inventario ASI, la subescala manejo de impresiones del BIDR-16 y un conjunto de preguntas sobre datos sociodemográficos (i.e., género, edad, nivel educativo y nacionalidad). Un mensaje al inicio del formulario informaba acerca de los objetivos del estudio. Después los participantes debían dar su consentimiento informado y aceptar participar en el estudio para pasar a completar el formulario en línea. La participación fue anónima.
Los datos se obtuvieron a través de un muestreo en línea, difundiendo el formulario por medio de las redes sociales. También se contó con la colaboración del Consejo Nacional para la Igualdad de Género de Ecuador, entidad estatal que difundió el formulario a través de sus cuentas oficiales en redes sociales. Los datos se recogieron de octubre a diciembre de 2019.

\section{Análisis de datos}

Con el objetivo de adaptar y validar la escala A-IPVAW a la población ecuatoriana, se llevaron a cabo los siguientes análisis. En primer lugar, se calcularon los estadísticos descriptivos de los ítems (media, desviación típica, asimetría, y kurtosis), así como el índice de homogeneidad corregido, que expresa la correlación entre cada ítem y el resto de la escala.

A continuación, se llevó a cabo un análisis factorial confirmatorio (AFC) replicando la solución unifactorial de la escala original (MartínFernández et al., 2018). Se utilizó para ello el método de estimación de mínimos cuadrados ponderados con medias y varianzas ajustadas (WLSMV), pues este procedimiento resulta especialmente adecuado cuando los datos son ordinales ( $\mathrm{Li}, 2016)$. La bondad de ajuste se evaluó utilizando una combinación de índices de ajuste, indicando valores del CFI y TLI iguales o superiores a .95 un buen ajuste, valores del RMSEA inferiores a .06 o a .08 un ajuste bueno o mediocre, respectivamente y valores del SRMR inferiores a .08 un ajuste adecuado (Hu \& Bentler, 1999). Una vez determinada la estructura latente de la escala, se examinó su consistencia interna a través de los estadísticos $\alpha$ de Cronbach y del $\omega$ de McDonald.

Se evaluó en tercer lugar la posible carga de deseabilidad social de cada ítem, realizando para ello un nuevo AFC utilizando los ítems de manejo de impresiones del BIDR-16 como marcadores (Ferrando, 2005). Se estimó para ello un modelo en el que todos los ítems de la escala A-IPVAW saturaban en un factor específico de actitudes de aceptabilidad de la VPM, y a la vez en un factor general de deseabilidad social en el que también saturan los ítems del BIDR-16. Este modelo es ortogonal, de forma que los factores no están relacionados entre sí, y es así posible determinar el porcentaje de varianza común explicada por los 
Tabla 1. Estadísticos descriptivos de los ítems de la escala A-IPVAW

\begin{tabular}{llllcr}
\hline & $\mathrm{M}$ & SD & Asimetría & Kurtosis & rítem-test \\
\hline a1 & 1.22 & .47 & $1.96(.01)$ & $3.09(.01)$ & .45 \\
a2 & 1.33 & .56 & $1.47(.02)$ & $1.18(.02)$ & .41 \\
a3 & 1.23 & .50 & $2.12(.02)$ & $3.68(.02)$ & .50 \\
a4 & 1.32 & .57 & $1.62(.02)$ & $1.62(.2)$ & .51 \\
a5 & 1.09 & .34 & $3.96(.01)$ & $16.05(.01)$ & .65 \\
a6 & 1.21 & .48 & $2.27(.02)$ & $4.39(.02)$ & .63 \\
a7 & 1.07 & .30 & $4.60(.01)$ & $22.07(.01)$ & .80 \\
a8 & 1.07 & .30 & $4.55(.01)$ & $21.63(.01)$ & .80 \\
a9 & 1.07 & .31 & $4.60(.01)$ & $21.91(.01)$ & .79 \\
a10 & 1.06 & .29 & $5.00(.01)$ & $26.16(.01)$ & .81 \\
a11 & 1.07 & .30 & $4.64(.01)$ & $22.53(.01)$ & .83 \\
a12 & 1.07 & .29 & $4.41(.01)$ & $2.50(.01)$ & .80 \\
a13 & 1.10 & .36 & $3.76(.01)$ & $14.26(.01)$ & .71 \\
a14 & 1.08 & .34 & $4.27(.01)$ & $18.46(.01)$ & .72 \\
a15 & 1.15 & .40 & $2.73(.01)$ & $7.06(.01)$ & .62 \\
a16 & .31 & $4.06(.01)$ & $17.23(.01)$ & .73 \\
a17 & 1.08 & .40 & $2.92(.01)$ & $8.26(.01)$ & .60 \\
a18 & 1.14 & .34 & $3.79(.01)$ & $14.73(.01)$ & .65 \\
a19 & 1.09 & .25 & $5.54(.01)$ & $33.02(.01)$ & .84 \\
a20 & 1.05 & .36 & $3.14(.01)$ & $9.81(.01)$ & .60 \\
\hline
\end{tabular}

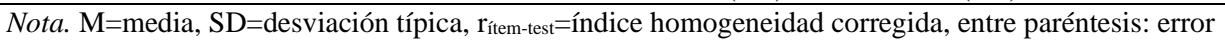
típico de los estadísticos de asimetría y kurtosis.

ítems de la escala A-IPVAW en el factor general de deseabilidad social. Además, si alguno de los ítems de la escala A-IPVAW presentara una saturación factorial superior a las del factor general de deseabilidad social, se consideraría que ese ítem está sesgado y debería eliminarse de la escala.

En cuarto lugar, se realizó un análisis de invarianza factorial de la escala entre géneros mediante un AFC multi-grupo (Putnick \& Bornstein, 2016). Con el objeto de prevenir un posible sesgo por capitalización del azar, se utilizó una segunda muestra para este análisis, pues de emplearse la misma muestra se estaría sobrestimando la bondad de ajuste de los modelos puestos a prueba. Se estimaron tres modelos anidados, evaluando secuencialmente la invarianza configural, escalar y métrica de la escala A-IPVAW. El modelo de invarianza configural aplica el mismo modelo factorial tanto para hombres como para mujeres, dejando libres el resto de parámetros. El modelo de invarianza métrica, por su parte, además de aplicar el mismo modelo, añade una restricción de igualdad para las saturaciones factoriales entre grupos, de manera que estas tomen el mismo valor entre hombres y mujeres. El modelo de invarianza escalar va un paso más allá y, además de la restricción de igualdad para las saturaciones factoriales, también la aplica para los umbrales de los ítems, fijando al mismo valor estos parámetros para ambos géneros. Para evaluar el ajuste de estos modelos, se siguieron las pautas de Cheung y Rensvold
(2002), comparando el ajuste de los modelos utilizando los puntos de corte para los índices de ajuste sugeridos por Meade et al. (2008; $\Delta \mathrm{CFI} \leq .002, \Delta \mathrm{RMSEA} \leq .007$ ), que funcionan mejor con indicadores ordinales (Sass et al., 2014).

Finalmente, se examinaron las evidencias de validez de la escala basadas en su relación con otros constructos actitudinales teóricamente relacionados con la aceptabilidad como son las actitudes de culpabilización de la víctima en casos de VPM, la severidad percibida de la VPM, y el sexismo hostil y benevolente. Se compararon también las puntuaciones de la escala en función del sexo, la edad, y el nivel de estudios.

Los análisis de este estudio se llevaron a cabo con la librería psych del programa estadístico $\mathrm{R}(\mathrm{R}$ Core Team, 2019; Revelle, 2012) y con el programa Mplus 8.2 (Muthén \& Muthén, 2017).

\section{Resultados}

\section{Análisis descriptivo}

Todos los ítems mostraron un promedio cercano a 1, con desviaciones típicas alrededor de .40 y elevados valores de asimetría y kurtosis (Tabla 1). Todo ello indica que las distribuciones de los ítems estuvieron marcadamente apuntadas y desplazadas hacia la izquierda, lo que implica que la mayoría de los participantes eligieron la categoría "nada aceptable" en casi todos los ítems. Los índices de homogeneidad corregidos fueron elevados, con valores superiores a .40 para todos 
los ítems, lo que indicó una estrecha relación entre cada ítem y el resto de la escala A-IPVAW.

\section{Análisis factorial confirmatorio y consistencia interna}

Dada la distribución asimétrica y leptokúrtica de los ítems, la estructura latente de la escala se examinó mediante un AFC estimando un modelo unifactorial con WLSMV. El ajuste del modelo resultante fue muy bueno (CFI=.97, TLI=.96, RMSEA $[95 \% \quad \mathrm{CI}]=.062 \quad[.057 ; \quad .062]$, SRMR=.058), y las saturaciones factoriales de los ítems fueron altas, con valores superiores a $.60 \mathrm{y}$ errores estándar de estimación bajos (alrededor de .03) (Figura 1). La consistencia interna de la escala fue muy buena $(\alpha=.93, \omega=.94)$.

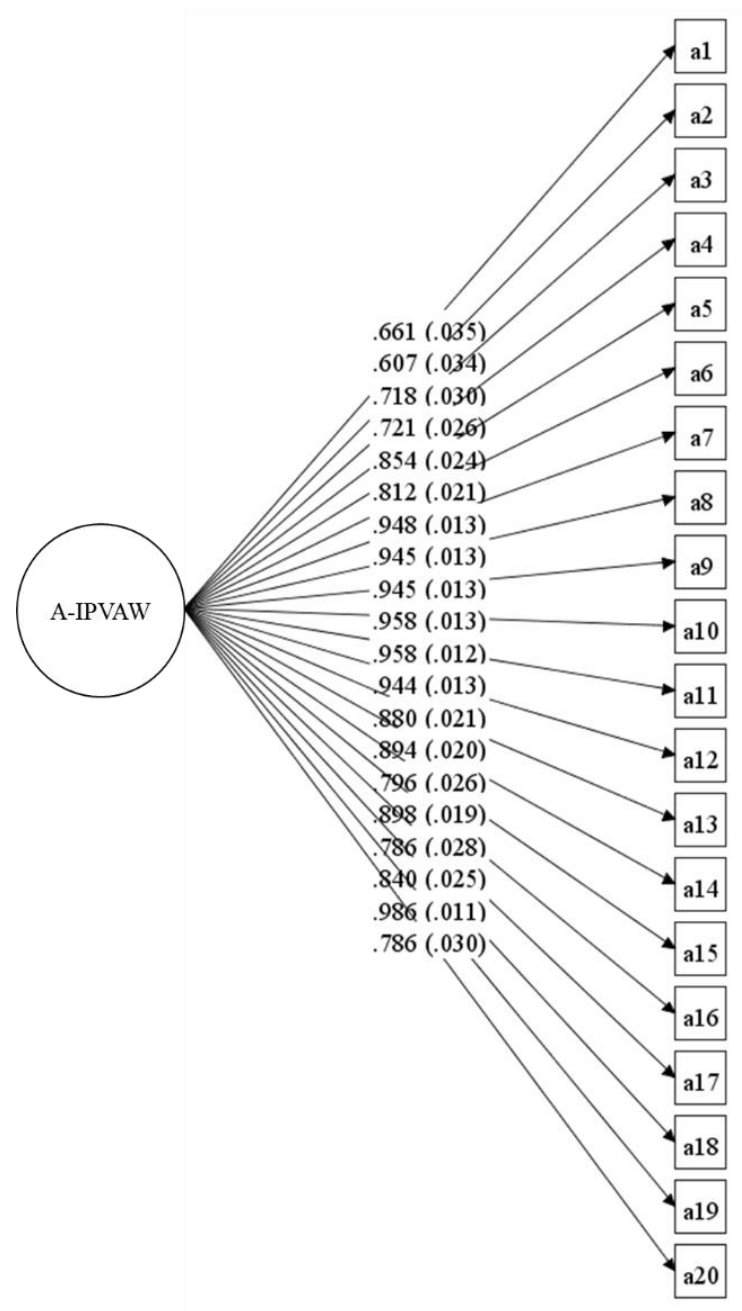

Figura 1. Modelo unifactorial de la escala

$$
\text { A-IPVAW }
$$

\section{Análisis de la deseabilidad social}

A continuación, se planteó un nuevo modelo en el que todos los ítems de la escala A-IPVAW saturaban en un factor de aceptabilidad de la VPM y en otro de deseabilidad social, en el que también saturaban los ítems de manejo de impresiones del BIDR-16. La bondad de ajuste de este modelo fue adecuada (CFI $=.95$, TLI=.94, RMSEA [95\% $\mathrm{CI}]=.060$ [.057; .064], SRMR=.078), y de nuevo, las saturaciones de los ítems de la escala fueron elevadas en el factor específico de aceptabilidad, presentando todos ellos valores superiores a .60 con errores bajos (alrededor de .03) (Tabla 2). Con respecto al factor general de deseabilidad social, se encontró que las saturaciones de los ítems de la escala A-IPVAW fueron bajas, tomando valores por debajo de $|0.20|$ en todos los casos. Los ítems de manejo de impresiones, sin embargo, mostraron saturaciones factoriales altas, alrededor de 0.60. El porcentaje de varianza común explicada por los ítems de la escala AIPVAW del factor general de deseabilidad social fue del $0.06 \%$, explicando los ítems del BIDR-16 el $99.94 \%$ restante, lo que permite descartar un posible sesgo por deseabilidad social en los ítems de la escala A-IPVAW.

Tabla 2. Saturaciones factoriales estandarizadas del modelo de deseabilidad social

\begin{tabular}{|c|c|c|c|c|}
\hline \multirow[t]{2}{*}{ Ítem } & \multicolumn{2}{|c|}{ Aceptabilidad VPM } & \multicolumn{2}{|c|}{$\begin{array}{c}\text { Deseabilidad } \\
\text { social } \\
\end{array}$} \\
\hline & $\lambda$ & S.E. & $\lambda$ & S.E. \\
\hline a1 & .67 & .04 & .10 & .05 \\
\hline a2 & .61 & .04 & .16 & .04 \\
\hline a3 & .72 & .03 & .07 & .05 \\
\hline $\mathrm{a} 4$ & .72 & .03 & .06 & .05 \\
\hline a5 & .85 & .03 & -.08 & .07 \\
\hline a6 & .82 & .02 & .04 & .05 \\
\hline a7 & .94 & .02 & -.14 & .08 \\
\hline a8 & .93 & .02 & -.18 & .09 \\
\hline a9 & .94 & .02 & -.10 & .08 \\
\hline a10 & .95 & .02 & -.11 & .08 \\
\hline a11 & .95 & .02 & -.16 & .08 \\
\hline a12 & .94 & .02 & -.12 & .08 \\
\hline a13 & .88 & .02 & -.06 & .07 \\
\hline a14 & .89 & .02 & -.11 & .08 \\
\hline a15 & .80 & .03 & .01 & .06 \\
\hline a16 & .90 & .02 & -.06 & .08 \\
\hline a17 & .79 & .03 & .03 & .06 \\
\hline a18 & .84 & .03 & -.01 & .07 \\
\hline a19 & .98 & .02 & -.11 & .10 \\
\hline a20 & .80 & .03 & .11 & .06 \\
\hline bidr1 & & & .64 & .02 \\
\hline bidr2 & & & .65 & .02 \\
\hline bidr3 & & & .58 & .02 \\
\hline bidr4 & & & .67 & .02 \\
\hline bidr5 & & & .59 & .02 \\
\hline bidr6 & & & .56 & .03 \\
\hline bidr7 & & & .66 & .02 \\
\hline bidr8 & & & .74 & .02 \\
\hline
\end{tabular}


Tabla 3. Bondad de ajuste de los modelos de invarianza factorial

\begin{tabular}{lrrrr}
\hline & $\chi^{2}$ & gl & CFI & RMSEA (95\% IC) \\
\hline Invarianza & & & & \\
Configural & 537.75 & 340 & .985 & $.058(.049 ; .067)$ \\
Métrica & 529.67 & 359 & .987 & $.052(.043 ; .062)$ \\
\multicolumn{1}{l}{ Escalar } & 592.62 & 399 & .985 & $.053(.044 ; .062)$ \\
\hline $\begin{array}{l}\text { Nota. } \text { gl=grados de libertad, CFI=índice comparativo de } \\
\text { ajuste, RMSEA=Raíz del error medio cuadrático ajustada. }\end{array}$
\end{tabular}

\section{Invarianza factorial}

Se empleó una segunda muestra para llevar a cabo los análisis de invarianza factorial entre géneros. Los resultados mostraron un buen ajuste para los modelos de invarianza configural, métrica y escalar (Tabla 3). Además, al añadir la restricción de igualdad para las saturaciones factoriales y para los umbrales de los ítems entre hombres y mujeres, el ajuste del modelo no empeoró sustantivamente $(\triangle \mathrm{CFI}=.002$, $\triangle \mathrm{RMSEA}=.006$ y $\Delta \mathrm{CFI}=.002, \Delta \mathrm{RMSEA}=.001$, respectivamente), lo que permite establecer que la escala A-IPVAW es invariante entre géneros.

\section{Otras evidencias de validez}

Para evaluar las evidencias de validez de la escala basadas en sus relaciones con otras variables, se obtuvieron las correlaciones entre las puntuaciones de la escala A-IPVAW con las escalas VB-IPVAW, PS-IPVAW, y el ASI (Tabla 4). Se encontró que los participantes con mayores niveles de aceptabilidad de la VPM presentaban a su vez mayores niveles de actitudes de culpabilización de la víctima, de sexismo hostil y benevolente y menores niveles de gravedad percibida de la VPM.

Tabla 4. Correlaciones entre la escala A-IPVAW

\begin{tabular}{|c|c|c|c|c|}
\hline & $\begin{array}{c}\text { VB- } \\
\text { IPVAW }\end{array}$ & $\begin{array}{c}\text { PS- } \\
\text { IPVAW }\end{array}$ & $\begin{array}{c}\text { Sexismo } \\
\text { Hostil }\end{array}$ & $\begin{array}{c}\text { Sexismo } \\
\text { Benevolente }\end{array}$ \\
\hline A-IPVAW & $.40^{* * *}$ & $-.29^{* *}$ & $.28^{* *}$ & $.17^{* *}$ \\
\hline VB-IPVAW & & $-.20^{* * *}$ & $.28^{* *}$ & $.21^{* *}$ \\
\hline PS-IPVAW & & & $-.09^{*}$ & -.01 \\
\hline $\begin{array}{l}\text { Sexismo } \\
\text { Hostil }\end{array}$ & & & & $.21^{* *}$ \\
\hline $\begin{array}{l}\text { Nota. A-IPVA } \\
\text { VPM. VB-IP } \\
\text { la víctima en } \\
\text { percibida de } 1\end{array}$ & $\begin{array}{l}\text { V: escala } \\
\text { iW: escal } \\
\text { sos de VP } \\
\text { JPM. *:p }\end{array}$ & $\begin{array}{l}\text { de actitud } \\
\text { a de actit } \\
\text { M. PS-If } \\
<.01 .^{* *} \text { : }\end{array}$ & $\begin{array}{l}\text { de ace } \\
\text { es de cl } \\
\text { AW: es } \\
001 .\end{array}$ & $\begin{array}{l}\text { ilidad de la } \\
\text { bilización de } \\
\text { a de severidad }\end{array}$ \\
\hline
\end{tabular}

Finalmente se realizaron comparaciones entre las puntuaciones de los participantes en la escala en función del género, el nivel de estudios y la edad a través de un ANOVA. Se encontraron diferencias significativas entre hombres $(M=.30$, $S D=.84)$ y mujeres $(\mathrm{M}=.04, \mathrm{SD}=.75)$ con un tamaño del efecto moderado $(F[1]=18.55$, $\left.p<.001, \eta^{2}=.04\right)$, mostrando las mujeres niveles de aceptabilidad menores que los hombres. Se hallaron asimismo diferencias significativas en función del nivel de estudios con un tamaño del efecto pequeño $\left(F[3]=4.97, p<.001, \eta^{2}=.02\right)$. Las pruebas post-hoc revelaron que las diferencias se encontraron entre los participantes con formación universitaria, quienes mostraron niveles más bajos de aceptabilidad, y el resto de niveles educativos $\left(M_{\text {básica }}=.42, \quad S D_{\text {básica }}=.82 ; \quad M_{\text {secundaria }}=.26\right.$, $S D_{\text {secundaria }}=.83 ; \quad M_{\text {estudiante }}$ universitario $=.21, S D_{\text {estudiante }}$ universitario $=.82 ;$ Muniversitaria $=-.01, S D_{\text {universitaria }}=.73$ ).

Con respecto a la edad, se encontró una correlación negativa entre la aceptabilidad de la VPM y la edad ( $r=-.12, p<.001)$, de manera que los participantes más jóvenes tendieron a mostrar niveles de aceptabilidad más bajos que las personas de mayor edad.

\section{Discusión}

Ecuador es uno de los países latinoamericanos con una mayor proporción de casos de VPM. Las actitudes hacia la VPM son cada vez más reconocidas como un factor esencial para comprender mejor este importante problema social y desarrollar iniciativas de prevención e intervención eficaces (Gracia et al., 2020). Por ello, se hace indispensable contar con instrumentos con evidencias de fiabilidad y validez que permitan evaluar de manera consistente la aceptabilidad de la VPM en este contexto sociocultural (Villagrán et al., 2020).

En este estudio se presenta la validación de la escala de actitudes de aceptabilidad de la VPM (A-IPVAW) en población ecuatoriana. Los resultados muestran que esta medida posee una buena consistencia interna, que es invariante entre géneros y que sus ítems tienen una baja carga de deseabilidad social. Además, se relaciona con otros constructos relevantes como el sexismo ambivalente, las actitudes de culpabilización de la 
víctima y una menor gravedad percibida de la VPM, así como con variables sociodemográficas que indican que la aceptabilidad de la VPM es más frecuente en personas del sexo masculino, con menor nivel de estudios y mayor edad. Estos hallazgos proporcionan evidencia de que la escala A-IPVAW es un instrumento con suficientes evidencias de fiabilidad y validez para evaluar la aceptabilidad de la VPM en el contexto sociocultural de Ecuador.

En relación a la estructura latente y fiabilidad, esta medida mantiene la estructura factorial de la escala original y una muy buena consistencia interna. Esto indica que este instrumento es capaz de evaluar con precisión las actitudes de aceptabilidad de la VPM, respetando la conceptualización original del constructo. Acerca de las diferencias de género, se consiguió establecer un nivel de invarianza escalar entre hombres y mujeres, lo que asegura que ambos grupos interpretan todos los ítems de manera similar y permite hacer comparaciones entre géneros, resolviendo una de las limitaciones que presentaba la escala original (Martín-Fernández et al., 2018).

En cuanto al análisis del sesgo de deseabilidad social, constituye unas de las principales fortalezas de este estudio, ya que se realiza por primera vez con este instrumento. Este sesgo constituye una de las principales amenazas a la validez de contenido de cualquier escala autoinformada que evalúa temas socialmente sensibles, como los relacionados con la VPM (Grimm, 2010). Los resultados ponen de manifiesto que los ítems de la escala A-IPVAW tienen una baja carga de deseabilidad social, explicando tan sólo el $0.06 \%$ de la varianza común del factor general de deseabilidad, lo que permite descartar que los participantes hayan respondido a los ítems de acuerdo a este sesgo de respuesta.

Con respecto a las evidencias de validez basadas en la relación de la escala con otras variables, se encontró que las actitudes de aceptabilidad de la VPM se relacionan positivamente con el sexismo hostil y benevolente. Esto sugiere que las personas que consideran a la mujer como inferior al hombre o necesitada de su protección (Glick \& Fiske, 1996), son a su vez más propensas a presentar niveles más altos de aceptabilidad de la VPM. Estos resultados van en línea con investigaciones previas que consideran el sexismo como un posible facilitador de actitudes legitimadoras de la VPM (Glick et al., 2002; Gracia et al., 2018; Herrera et al., 2012; Herrero et al., 2017; MartínFernández et al., 2018).

Se encontró que las actitudes de aceptabilidad de la VPM se relacionan positivamente con las actitudes de culpabilización de la víctima. Es decir, aquellas personas que atribuyen la causa de la VPM a las víctimas son también más propensas a considerar los incidentes de violencia como aceptables, lo que también es consistente con estudios previos (Gracia et al., 2018; Gracia \& Tomás, 2014; Martín-Fernández et al., 2018; Valor-Segura et al., 2011).

Se halló asimismo una relación negativa entre las actitudes de aceptabilidad y la gravedad percibida de la VPM. Estos resultados van en línea con investigaciones anteriores que también sugieren que la aceptabilidad de la VPM está relacionada con la gravedad que las personas atribuyen a distintos escenarios de la VPM. Cuando un suceso violento es percibido como menos grave tiende a su vez a evaluarse como más aceptable, lo que puede llevar a justificar o incluso alentar la VPM (Gracia et al., 2015; Martín-Fernández et al., 2018; Taylor \& Sorenson, 2005).

Con relación a las variables sociodemográficas, nuestros resultados mostraron diferencias significativas por género, edad y nivel educativo. En cuanto al género, se encontró un tamaño del efecto moderado que mostró que los hombres presentan en promedio niveles de actitudes de aceptabilidad de la VPM mayores que las mujeres, lo que es consistente con la literatura previa (Carlson \& Worden, 2005; Fincham et al., 2008; Gracia et al., 2015; Gracia \& Tomás, 2014; Martín-Fernández et al., 2018), y corrobora que el género es uno de los predictores más consistentes de las actitudes públicas hacia la VPM (Flood \& Pease, 2009).

Con respecto a la edad y el nivel educativo, se encontró un tamaño del efecto pequeño que sugiere que las personas de mayor edad y con menor nivel educativo presentan niveles de aceptabilidad más altos que las personas más jóvenes y con mayor educación, resultados que 
van en línea con otros estudios (Carlson \& Worden, 2005; Coop et al., 2019; Flood \& Pease, 2009; Gracia \& Tomás, 2014; Safranoff, 2017; Worden \& Carlson, 2005).

Por último, este estudio tiene limitaciones y fortalezas. Con respecto a sus limitaciones, hay que señalar que si bien la estrategia de muestreo en línea es eficaz y rentable (Thornton et al., 2016), presenta mayores dificultades en la verificación de la información sociodemográfica de los participantes si se compara con estrategias de muestreo más tradicionales (Topolovec-Vranic \& Natarajan, 2016). El sesgo de autoselección en la muestra es otra limitación, puesto que los individuos que aceptaron ser parte del estudio podrían estar más motivados que el resto de la población. Asimismo, al ser un estudio transversal no permite examinar cómo evolucionan las actitudes de aceptabilidad de la VPM con el tiempo.

En cuanto a sus fortalezas, la validación de la escala española A-IPVAW en población ecuatoriana resuelve algunas de las limitaciones de la escala original al establecer un nivel de invarianza escalar entre hombres y mujeres, demostrar la baja carga de deseabilidad social que posee el instrumento y validarlo en un contexto sociocultural diferente, abriendo una puerta para la realización de futuros estudios interculturales sobre las actitudes hacia la VPM.

Contar con un instrumento con evidencias de fiabilidad y validez para evaluar la aceptabilidad de la VPM en el contexto sociocultural de Ecuador no solo es teóricamente importante, sino que tiene implicaciones prácticas. Debido a la relación que existe entre la aceptabilidad de la VPM y su perpetración (Coop et al., 2019; Fleming et al., 2015; Gracia et al., 2015; MartínFernández et al., 2018; Romero-Martinez et al., 2019; Waltermaurer, 2012), esta escala podría utilzarse para evaluar la efectividad de programas de intervención con hombres perpetradores de VPM, monitorizando el cambio de sus actitudes (Arce et al., 2020; Carbajosa et al., 2017; Lila et al., 2018; Martín-Fernández et al., 2018; Santirso et al., 2020).

También es un instrumento útil en términos de prevención, puesto que haría posible identificar las actitudes de aceptabilidad de la VPM en diferentes grupos (e.g., minorías étnicas, personal de salud, estudiantes, etc.) y en determinados contextos (e.g., universidades, vecindarios, zonas rurales, etc.). Como señalan Gracia et al. (2020), esto facilitaría el diseño y evaluación de campañas educativas focalizadas, orientadas a disminuir la aceptabilidad de la VPM y promover la ayuda hacia las víctimas, lo que permitiría, en definitiva, optimizar recursos y contribuir de manera más eficaz a la disuasión y control colectivo de la VPM en el contexto sociocultural ecuatoriano.

\section{Referencias}

Arce, R., Arias, E., Novo, M., \& Fariña, F. (2020). Are interventions with batterers effective? A meta-analytical review. Psychosocial Intervention, 29(3), 153-164. https://doi.org/10.5093/pi2020a11

Barker, G., Contreras, J. M., Heilman, B., Singh, A. K., Verma, R. K., \& Nascimento, M. (2011). Evolving men. Initial Results from the International Men and Gender Equality Survey (IMAGES). ICRW.

https://www.icrw.org/wpcontent/uploads/2016/10/Evolving-MenInitial-Results-from-the-International-Menand-Gender-Equality-Survey-IMAGES-1.pdf

Bott, S., Guedes, A., Goodwin, M., \& Adams, J. (2013). Violence against women in Latin America and the Caribbean: Summary report: A comparative analysis of population-based data from 22 countries. OPS. http://www.endvawnow.org/uploads/browser/ files/paho-vaw-exec-summ-spa.pdf

Bucheli, M., \& Rossi, M. (2016). Attitudes towards intimate partner violence against women in Latin America. Universidad de la República.

http://dx.doi.org/10.2139/ssrn.2857151

Camacho, G. (2014). La violencia de género contra las mujeres en el Ecuador: Análisis de los resultados de la Encuesta Nacional sobre Relaciones Familiares y Violencia de Género contra las Mujeres. Consejo Nacional para la Igualdad de Género.

https://oig.cepal.org/sites/default/files/violenci a_de_gnero_ecuador.pdf

Campbell, J. C., \& Manganello, J. (2006). Changing public attitudes as a prevention 
strategy to reduce intimate partner violence. Journal of Aggression, Maltreatment \& Trauma, 13, 13-40.

https://doi.org/10.1300/J146v13n03_02

Carbajosa, P., Catalá-Miñana, A., Lila, M., \& Gracia, E. (2017). Differences in treatment adherence, program completion, and recidivism among batterer subtypes. The European Journal of Psychology Applied to Legal Context, 9, 93-101. https://doi.org/10.1016/j.ejpal.2017.04.001

Carlson, B. E., \& Worden, A. P. (2005). Attitudes and beliefs about domestic violence: Results of a public opinion survey I. Definitions of domestic violence, criminal domestic violence, and prevalence. Journal of Interpersonal Violence, 20, 1197-1218. https://doi.org/10.1177/0886260505278530

Cheung, G. W., \& Rensvold, R. B. (2002). Evaluating goodness-of-fit indexes for testing measurement invariance. Structural Equation Modeling, 9, 233-255. https://doi.org/10.1207/S15328007SEM0902_5

Copp, J. E., Giordano, P. C., Longmore, M. A., \& Manning, W. D. (2019). The development of attitudes toward intimate partner violence: An examination of key correlates among a sample of young adults. Journal of Interpersonal Violence, 34(7), 1357-1387. https://doi.org/10.1177/0886260516651311

Devries, K. M., Mak, J. Y., García-Moreno, C., Petzold, M., Child, J. C., Falder, G., Lim, S., Bacchus, L. J., Engell, R. E., Rosenfeld, L., Pallitto, C., Vos, T., Abrahams, N., \& Watts, C. H. (2013). The global prevalence of intimate partner violence against women. Science, 340, 1527-1528. https://doi.org/10.1126/science.1240937

Ellsberg, M., Jansen, H. A., Heise, L., Watts, C. H., \& Garcia-Moreno, C. (2008). Intimate partner violence and women's physical and mental health in the WHO multi-country study on women's health and domestic violence: an observational study. The Lancet, 371, 1165-1172.

https://doi.org/10.1016/S01406736(08)60522-X

Expósito, F., Moya, M. C., \& Glick, P. (1998). Sexismo ambivalente: medición y correlatos. Revista de Psicología Social, 13, 159-169. https://doi.org/10.1174/021347498760350641

Ferrando, P. J. (2005). Factor analytic procedures for assessing social desirability in binary items. Multivariate Behavioral Research, 40, 331-349.

https://doi.org/10.1207/s15327906mbr4003_3

Fincham, F. D., Cui, M., Braithwaite, S., \& Pasley, K. (2008). Attitudes toward intimate partner violence in dating relationships. Psychological Assessment, 20, 260-269. http://psycnet.apa.org/doi/10.1037/10403590.20.3.260

Fleming, P. J., McCleary-Sills, J., Morton, M., Levtov, R., Heilman, B., \& Barker, G. (2015). Risk factors for men's lifetime perpetration of physical violence against intimate partners: Results from the international men and gender equality survey (IMAGES) in eight countries. Plos One, 10(3).

https://doi.org/10.1371/journal.pone.0118639

Flood, M., \& Pease, B. (2009). Factors influencing attitudes to violence against women. Trauma, Violence, \& Abuse, 10, 125142.

https://doi.org/10.1177/1524838009334131

García-Moreno, C., Zimmerman, C., MorrisGehring, A., Heise, L., Amin, A., Abrahams, N., ... Watts, C. (2015). Addressing violence against women: A call to action. The Lancet, 385, 1685-1695.

https://doi.org/10.1016/S01406736(14)61830-4

Glick, P., \& Fiske, S. T. (1996). The ambivalent sexism inventory: Differentiating hostile and benevolent sexism. Journal of Personality and Social Psychology, 70, 491-512. https://doi.org/10.1037/0022-3514.70.3.491

Glick, P., Sakalli-Ugurlu, N., Ferreira, M. C., \& Aguiar de Souza, M. (2002). Ambivalent sexism and attitudes toward wife abuse in Turkey and Brazil. Psychology of Women Quarterly, 26, 292-297. https://doi.org/10.1111/1471-6402.t01-100068

Gracia, E. (2014). Intimate partner violence against women and victim-blaming attitudes among Europeans. Bulletin of the World Health Organization, 92, 380-381. https://doi.org/10.2471/BLT.13.131391 
Gracia, E., García, F., \& Lila, M. (2008). Police involvement in cases of intimate partner violence against women: The influence of perceived severity and personal responsibility. Violence Against Women, 14, 697-714. https://doi.org/10.1177/1077801208317288

Gracia, E., García, F., \& Lila, M. (2011). Police attitudes toward policing partner violence against women: Do they correspond to different psychosocial profiles?. Journal of Interpersonal Violence, 26(1), 189-207. http://dx.doi.org/10.1177/0886260510362892

Gracia, E., García, F., \& Lila, M. (2014). Male police officers' law enforcement preferences in cases of intimate partner violence versus non-intimate interpersonal violence: Do sexist attitudes and empathy matter? Criminal Justice and Behavior, 41(10), 1195-1213. https://doi.org/10.1177/0093854814541655

Gracia, E., Rodriguez, C. M., Martín-Fernández, M., \& Lila, M. (2020). Acceptability of family violence: Underlying ties between intimate partner violence and child abuse. Journal of Interpersonal Violence, 35(17-18), 32173236. https://doi.org/10.1177/0886260517707310

Gracia, E., Lila, M., \& Santirso, F. A. (2020). Attitudes toward intimate partner violence against women in the European Union. European Psychologist, 25, 104-121. https://doi.org/10.1027/1016-9040/a000392

Gracia, E., Martín-Fernández, M., Marco, M., Santirso, F. A., Vargas, V., \& Lila, M. (2018). The Willingness to Intervene in cases of Intimate Partner Violence Against Women (WI-IPVAW) Scale: Development and validation of the long and short versions. Frontiers in Psychology, 9, 1146. https://doi.org/10.3389/fpsyg.2018.01146

Gracia, E., Rodriguez, C. M., \& Lila, M. (2015). Preliminary evaluation of an analog procedure to assess acceptability of intimate partner violence against women: The Partner Violence Acceptability Movie Task. Frontiers in Psychology, 6, 1567.

https://doi.org/10.3389/fpsyg.2015.01567

Gracia, E., \& Tomás, J. M. (2014). Correlates of victim-blaming attitudes regarding partner violence against women among the Spanish general population. Violence Against Women,
20, 26-41.

https://doi.org/10.1177/1077801213520577

Grimm, P. (2010). Social desirability bias. Wiley international encyclopedia of marketing. https://doi.org/10.1002/9781444316568.wiem 02057

Guedes, A., García-Moreno, C., \& Bott, S. (2014). Violencia contra las mujeres en Latinoamérica y el Caribe. Foreign Affairs Latinoamérica, 14(1), 41-48.

https://www.paho.org/hq/dmdocuments/2014/ Foreign-Affairs-2014-Guedes-et-al-Violenciacontra-las-mujeres-en-LAC.pdf

Hart, C. M., Ritchie, T. D., Hepper, E. G., \& Gebauer, J. E. (2015). The balanced inventory of desirable responding short form (BIDR16). Sage Open, 5(4). https://doi.org/10.1177/2158244015621113

Heise, L. (2011). What works to prevent partner violence? An evidence overview. STRIVE. https://researchonline.1shtm.ac.uk/id/eprint/21 062/

Herrera, M. C., Expósito, F., \& Moya, M. (2012). Negative reactions of men to the loss of power in gender relations: Lilith vs. Eve. The European Journal of Psychology Applied to Legal Context, 4, 17-42.

https://search.proquest.com/docview/1140194 253 ? accountid $=14777$

Herrero, J., Rodríguez-Franco, L., RejanoHernández, L., Juarros-Basterretxea, J., \& Rodríguez-Díaz, F. J. (2020). The actorpartner interdependence model in the study of aggression and victimization within couples: An empirical examination in 361 dyads. Psychosocial Intervention, 29(3), 165-174. https://doi.org/10.5093/pi2020a12

Herrero, J., Rodríguez, F. J., \& Torres, A. (2017). Acceptability of partner violence in 51 societies: The role of sexism and attitudes toward violence in social relationships. Violence Against Women, 23, 351-367. https://doi.org/10.1177/1077801216642870

Hu L. T., \& Bentler P. M. (1999). Cutoff criteria for fit indices in covariance structure analysis: Conventional criteria versus new alternatives. Structural Equation Modeling, 6, 1-55. https://doi.org/10.1080/10705519909540118

Instituto Nacional de Estadísticas y Censos (2019). Encuesta nacional sobre relaciones 
familiares y violencia de género contra las mujeres.

https://www.ecuadorencifras.gob.ec/document os/web-

inec/Estadisticas_Sociales/Violencia_de_gene ro_2019/Boletin_Tecnico_ENVIGMU.pdf

Jewkes, R., Flood, M., \& Lang, J. (2015). From work with men and boys to changes of social norms and reduction of inequities in gender relations: A conceptual shift in prevention of violence against women and girls. The Lancet, $385,1580-1589$.

https://doi.org/10.1016/S01406736(14)61683-4

Kogut, T. (2011). Someone to blame: When identifying a victim decreases helping. Journal of Experimental Social Psychology, 47(4), 748-755. https://doi.org/10.1016/j.jesp.2011.02.011

Li, C. H. (2016). Confirmatory factor analysis with ordinal data: Comparing robust maximum likelihood and diagonally weighted least squares. Behavior Research Methods, 48, 936-949. https://doi.org/10.3758/s13428-015-0619-7

Lila, M., Gracia, E., \& Catalá-Miñana, A. (2018). Individualized motivational plans in batterer intervention programs: A randomized clinical trial. Journal of Consulting and Clinical Psychology, 86(4), 309-320.

https://doi.org/10.1037/ccp0000291

Lila, M., Gracia, E., \& Garcia, F. (2010). Police attitudes toward intervention in cases of partner violence against women: The influence of sexism and empathy. Revista de Psicología Social, 25(3), 313-323. https://doi.org/10.1174/021347410792675570

Lila, M., Gracia, E., \& Garcia, F. (2013). Ambivalent sexism, empathy and law enforcement attitudes towards partner violence against women among male police officers. Psychology, Crime \& Law, 19(10), 907-919. https://doi.org/10.1080/1068316X.2012.719619

Lila, M., Gracia, E., \& Murgui, S. (2013). Psychological adjustment and victim-blaming among intimate partner violence offenders: The role of social support and stressful life events. The European Journal of Psychology Applied to Legal Context, 5, 147-153. https://doi.org/10.5093/ejpalc2013a4

Lila, M., Oliver, A., Catalá-Miñana, A., Galiana, L., \& Gracia, E. (2014). The intimate partner violence responsibility attribution scale (IPVRAS). The European Journal of Psychology Applied to Legal Context, 6, 2936. https://doi.org/10.5093/ejpalc2014a4

López-Cepero Borrego, J., Rodríguez Franco, L., \& Rodríguez Díaz, F. J. (2015). Evaluación de la violencia de pareja. Una revisión de instrumentos de evaluación conductual. Revista Iberoamericana de Diagnóstico y Evaluación - e Avaliação Psicológica, 2(40), 37-50.

Martín-Fernández, M., Gracia, E., \& Lila, M. (2018). Assessing victim-blaming attitudes in cases of intimate partner violence against women: Development and validation of the VB-IPVAW scale. Psychosocial Intervention, 27, 133-143.

https://doi.org/10.5093/pi2018a18

Martín-Fernández, M., Gracia, E., \& Lila, M. (2019). Psychological intimate partner violence against women in the European Union: A cross-national invariance study. BMC Public Health, 19, 1739. https://doi.org/10.1186/s12889-019-7998-0

Martín-Fernández, M., Gracia, E., \& Lila, M. (2020). Ensuring the comparability of crossnational survey data on intimate partner violence against women: A cross-sectional, population-based study in the European Union. BMJ Open, 10(3), e032231. http://dx.doi.org/10.1136/bmjopen-2019032231

Martín-Fernández, M., Gracia, E., Marco, M., Vargas, V., Santirso, F. A., \& Lila, M. (2018). Measuring acceptability of intimate partner violence against women: Development and validation of the A-IPVAW scale. The European Journal of Psychology Applied to Legal Context, 10(1), 26-34. https://doi.org/10.5093/ejpalc2018a3

Meade, A. W., Johnson, E. C., \& Braddy, P. W. (2008). Power and sensitivity of alternative fit indices in tests of measurement invariance. Journal of Applied Psychology, 93, 568-592.

https://doi.org/10.1037/0021-9010.93.3.568 
Muthén, L.K. \& Muthén, B.O. (2017). Mplus user's guide (8th edition). Muthén \& Muthén. https://www.statmodel.com/download/usersgu ide/MplusUserGuideVer_8.pdf

Organización Mundial de la Salud (2013). Estimaciones mundiales y regionales de la violencia contra la mujer: prevalencia y efectos de la violencia conyugal y la violencia sexual no conyugal en la salud. http://apps.who.int/iris/bitstream/10665/85243 /1/WHO_RHR_HRP_13.06_spa.pdf

Putnick, D. L., \& Bornstein, M. H. (2016). Measurement invariance conventions and reporting: The state of the art and future directions for psychological research. Developmental Review, 41, 71-90. https://doi.org/10.1016/j.dr.2016.06.004

$\mathrm{R}$ Core Team (2019). R: A language and environment for statistical computing. $\mathrm{R}$ Foundation for Statistical Computing. https://www.R-project.org/

Revelle, W. (2018) psych: Procedures for personality and psychological research (Version 1.8.10). Northwestern University. https://CRAN.R-project.org/package=psych

Romero-Martínez, Á., Lila, M., Gracia, E., Rodriguez, C. M., \& Moya-Albiol, L. (2019). Acceptability of intimate partner violence among male offenders: The role of set-shifting and emotion decoding dysfunctions as cognitive risk factors. International Journal of Environmental Research and Public Health, 16(9), 1537.

https://doi.org/10.3390/ijerph16091537

Saccomano, C. (2017). El feminicidio en América Latina:¿vacío legal o déficit del Estado de derecho? Revista CIDOB d'Afers Internacionals, (117), 51-78. https://www.jstor.org/stable/26388133?seq=1

Sánchez-Hernández, M. D., Herrera-Enríquez, M. C., \& Expósito, F. (2020). Controlling behaviors in couple relationships in the digital age: Acceptability of gender violence, sexism, and myths about romantic love. Psychosocial Intervention, 29(2), 67-81. http://dx.doi.org/10.5093/pi2020a1

Sánchez-Prada, A., Delgado-Álvarez, C., BoschFiol, E., \& Ferrer-Pérez, V. A. (2019). Aportaciones sobre la Medición de Creencias acerca del Maltrato a la Mujer (IBWB) en
Población Española. Revista Iberoamericana de Diagnóstico y Evaluación - e Avaliação Psicológica, 4(53), 49-62.

https://doi.org/10.21865/RIDEP53.4.04

Santirso, F. A., Gilchrist, G., Lila, M., \& Gracia, E. (2020). Motivational strategies in interventions for intimate partner violence offenders: A systematic review and metaanalysis of randomized controlled trials. Psychosocial Intervention, 29(3), 175-190. https://doi.org/10.5093/pi2020a13

Safranoff, A. (2017). La aceptación de la violencia contra la mujer en la pareja: Un análisis comparativo de ocho países de América Latina. Anais, 1-18. http://www.abep.org.br/xxencontro/files/paper /621-352.pdf

Sass, D. A., Schmitt, T. A., \& Marsh, H. W. (2014). Evaluating model fit with ordered categorical data within a measurement invariance framework: A comparison of estimators. Structural Equation Modeling: A Multidisciplinary Journal, 21(2), 167-180. https://doi.org/10.1080/10705511.2014.882658

Stöckl, H., Devries, K., Rotstein, A., Abrahams, N., Campbell, J., Watts, C., \& GarcíaMoreno, C. (2013). The global prevalence of intimate partner homicide: A systematic review. The Lancet, 382, 859-865. https://doi.org/10.1016/S01406736(13)61030-2

Tausch, A. (2019). Multivariate analyses of the global acceptability rates of male intimate partner violence (IPV) against women based on World Values Survey data. The International Journal of Health Planning and Management, 34(4), 1155-1194.

https://doi.org/10.1002/hpm.2781

Taylor, C. A., \& Sorenson, S. B. (2005). Community-based norms about intimate partner violence: Putting attributions of fault and responsibility into context. Sex Roles, 53, 573-589.

http://dx.doi.org/10.1007/s11199-006-9155-3

Thornton, L., Batterham, P. J., Fassnacht, D. B., Kay-Lambkin, F., Calear, A. L., \& Hunt, S. (2016). Recruiting for health, medical or psychosocial research using Facebook: Systematic review. Internet Interventions, 4, 
72-81.

https://doi.org/10.1016/j.invent.2016.02.001

Topolovec-Vranic, J., \& Natarajan, K. (2016).

The use of social media in recruitment for medical research studies: A scoping review. Journal of Medical Internet Research, 18, e286. https://dx.doi.org/10.2196/jmir.5698

Valor-Segura, I., Expósito, F., \& Moya, M. (2011). Victim blaming and exoneration of the perpetrator in domestic violence: The role of beliefs in a just world and ambivalent sexism. The Spanish Journal of Psychology, 14, 195-206.

https://doi.org/10.5209/rev_SJOP.2011.v14.

$\mathrm{n} 1.17$

Vidal-Fernández, A., \& Megías, J. L. (2014). Attributions of blame to battered women when they are perceived as feminists or as "difficult to deal with". Spanish Journal of Psychology, 17. https://doi.org/10.1017/sjp.2014.26

Vilariño, M., Amado, B. G., Vázquez, M. J., \& Arce, R. (2018). Psychological harm in women victims of intimate partner violence: Epidemiology and quantification of injury in mental health markers. Psychosocial Intervention, 27(3), 145-152. http://dx.doi.org/10.5093/pi2018a23

Villagrán A. M., Martín-Fernández, M., Gracia, E., \& Lila, M. (2020). Adaptación y validación de la escala de actitudes de culpabilización de la víctima en casos de violencia de pareja contra la mujer (VBIPVAW) en población ecuatoriana. Revista Latinoamericana de Psicología, 52, 243-252. https://doi.org/10.14349/rlp.2020.v52.24

Waltermaurer, E. (2012). Public justification of intimate partner violence: A review of the literature. Trauma, Violence, \& Abuse, 13, 167-175. https://doi.org/10.1177/1524838012447699

Worden, A. P., \& Carlson, B. E. (2005). Attitudes and beliefs about domestic violence: Results of a public opinion survey: II. Beliefs about causes. Journal of Interpersonal Violence, 20(10), 1219-1243. https://doi.org/10.1177/0886260505278531 


\section{Anexo I}

Pienso que es aceptable para un hombre...

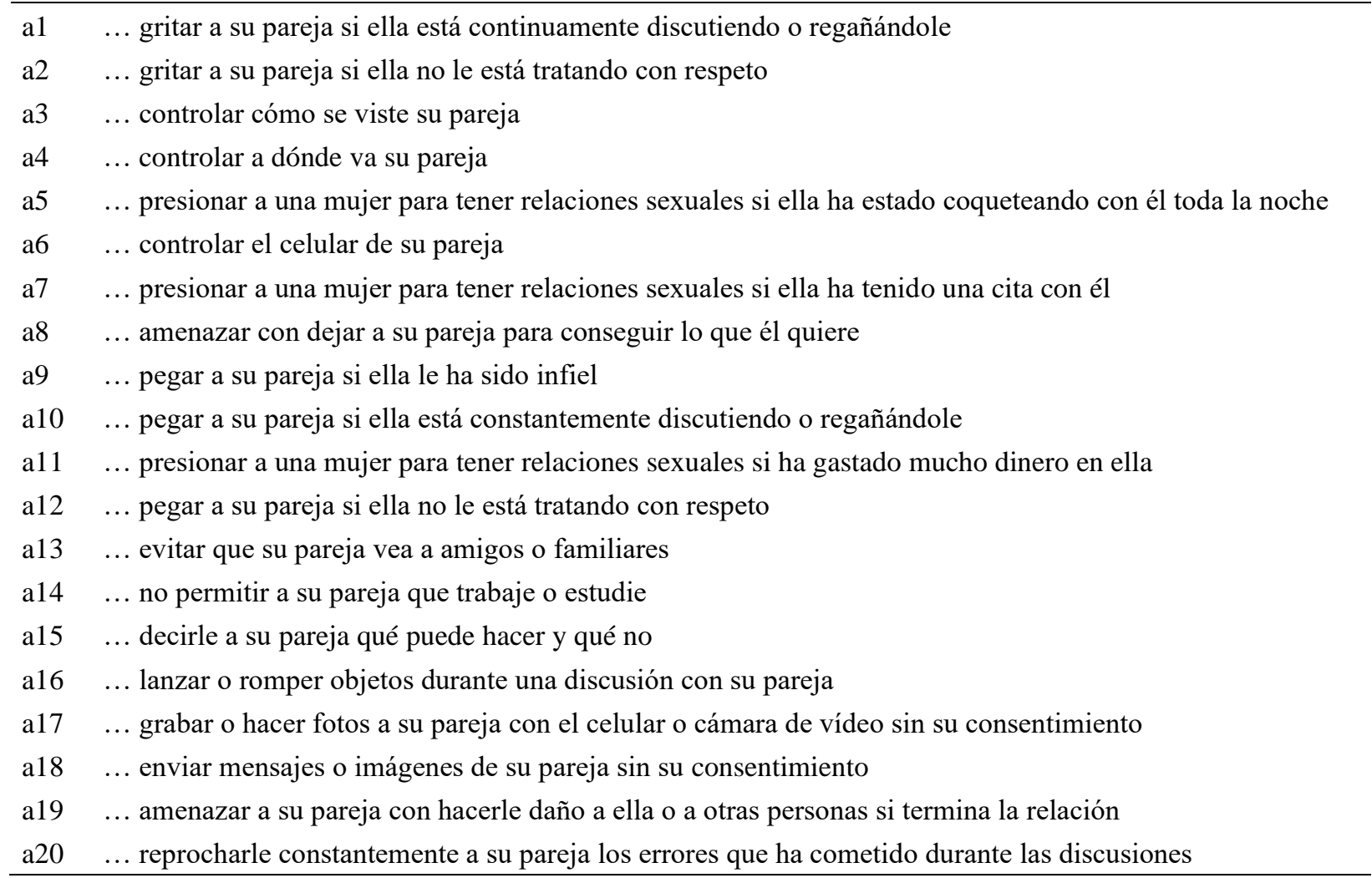
Nota. a=item de la escala de actitudes de aceptabilidad hacia la VPM (A-IPVAW). 